\title{
Resolving stellar populations using broad band photometry
}

\section{Maren Hempel ${ }^{1}$ \& Markus Kissler-Patig ${ }^{2}$}

\author{
${ }^{1}$ Department of Physics and Astronomy, Michigan State University, East Lansing, \\ MI 48824, USA \\ email: hempel@pa.msu.edu \\ ${ }^{2}$ European Southern Observatory, Karl-Schwarzschild Str.2, 85748 Garching, Germany \\ email: mkissler@eso.org
}

\begin{abstract}
Setting constraints on the evolutionary history of a galaxy requires informations about the age and metallicity of its stellar content. However, outside the Local Group of galaxies representative surveys on the age and metallicity of stellar populations in galaxies of different types (e.g. luminosity, environment) can only be performed with photometry. The latter requires special care in order to overcome the age- metallicity degeneracy of broad band colors. Combining optical and near-infrared observations of globular clusters is a powerful tool in this respect, using the different sensitivity of broad band colors regarding both parameters. Based on Single Stellar Population (SSP) models we derive the cumulative age distribution for various globular cluster systems and compare it to simulated systems with a known age distribution. So far this method has been tested successfully on various early-type galaxies, revealing a second generation of globular clusters in two galaxies in a sample of eight with convincing reliability.
\end{abstract}

Keywords. galaxies: formation, galaxies: evolution, star clusters, techniques: photometry

\section{Introduction}

To determine the age and metallicity of stellar populations in galaxies is crucial for our understanding of the underlying formation scenario. Fortunately, a specific formation scenario, e.g. hierarchical merging (e.g. Toomre \& Toomre 1972; Kauffmann, White \& Guiderdoni 1993), monolithic collapse (e.g. Chiosi \& Carraro 2000), or accretion (e.g. Hilker, Infante \& Richtler 1999) leaves its imprint in the age structure of the galaxy. The detection of different age populations within a galaxy will therefore allow us to set some constraints on its formation. This, however, requires the ability to disentangle stellar populations of different ages. Globular clusters represent Single Stellar Populations almost perfectly. They are found in almost every galaxy and can be observed at distances as far as $100 \mathrm{Mpc}$ (e.g. Coma galaxy cluster, Woodworth \& Harris 2000). Consequently, they are ideal tools to trace the major star formation events in their host galaxy, and we are now left with the task to detect globular cluster populations and to derive their relative ages. The method we present here is based on photometric data, allowing to observe representative globular cluster samples in each of the galaxies.

One of the features of globular cluster systems, which has to be explained by the formation/evolution of the host galaxy, is the bimodal color distribution, seemingly rather common in early-type galaxies (e.g. Gebhardt \& Kissler-Patig 1999; Kundu \& Whitmore 2001a; 2001b). Before we attempt to argue about the origin of the color distribution and its dependency of the galactocentric distance (see Figure 1) we need to know whether the different colors are due to age or metallicity differences. In order to lift the age-metallicity 


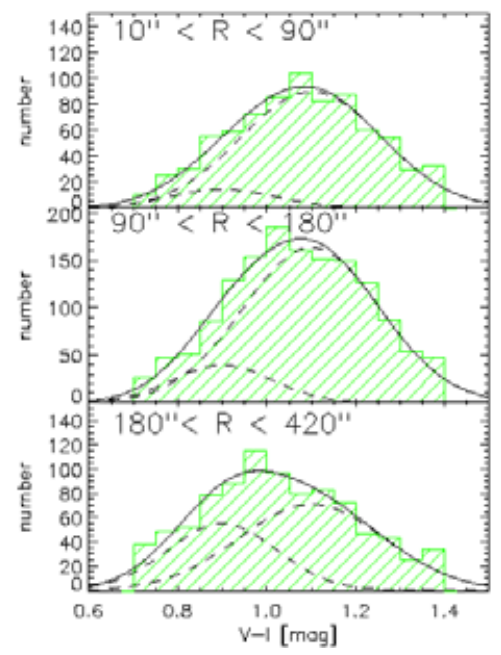

Figure 1. Radial color distribution in NGC 3311, the central cluster galaxy in the Hydra I cluster (see also Hilker 2003). The dashed lines in each plot refer to the two color populations, whereas the solid line represents their sum. The plots indicate the stronger concentration of the red population towards the center of the galaxy.

degeneracy of optical broad band colors (Worthey 1994) we combine optical, e.g. $(V-I)$, with infrared colors such as $(V-K)$ or $(V-H)$, taking advantage of the fact that the former are in comparison more age sensitive than infrared colors, which are dominated by the metallicity of the stellar populations.

\section{Data and Models}

To derive the relative ages of globular cluster sub-populations we combine optical and near -infrared photometric data. So far these were obtained with HST/WFPC2 (optical), and either VLT/ISAAC or HST/NICMOS2 in the near infrared. In order to probe the effect of external parameters, i.e. environment, or galaxy luminosity our galaxy sample contains cD galaxies (e.g. NGC 3311, M87), galaxies in groups (e.g. NGC 4365, NGC 5846, IC 4051) as well as rather isolated galaxies (e.g. NGC 7192, NGC 3115). The link to the galaxy formation is set via the probability for galaxy mergers (see Section 1), a formation scenario which not only allows but predicts bimodal color distributions in globular cluster systems. In Figure 2a we show an example of the final data set used for our analysis. The different isochrones (2 and 15 Gyr) show clearly the advantage of combined optical/near-infrared photometry with respect to the above mentioned agemetallicity degeneracy. However, this plot tells us also that the color of an individual globular cluster is not accurate enough to allow the determination of individual ages. Keeping in mind that at this point of the project we are mostly interested in detecting age sub-populations and to derive their relative ages we do not consider this to be a major drawback. A detailed description of how we infer the existence of globular cluster sub-populations differing in age by several Gyr can be found in Hempel \& Kissler-Patig (2004) and we will only describe the major steps here.

- Compare the optical color of the observed GCs with the predictions of Single Stellar Populations (e.g. Maraston 2005; Bruzual \& Charlot 2003; Vazdekis 1999) and select each GC into the corresponding age bin (see Figure $2 \mathrm{~b}$ ). 

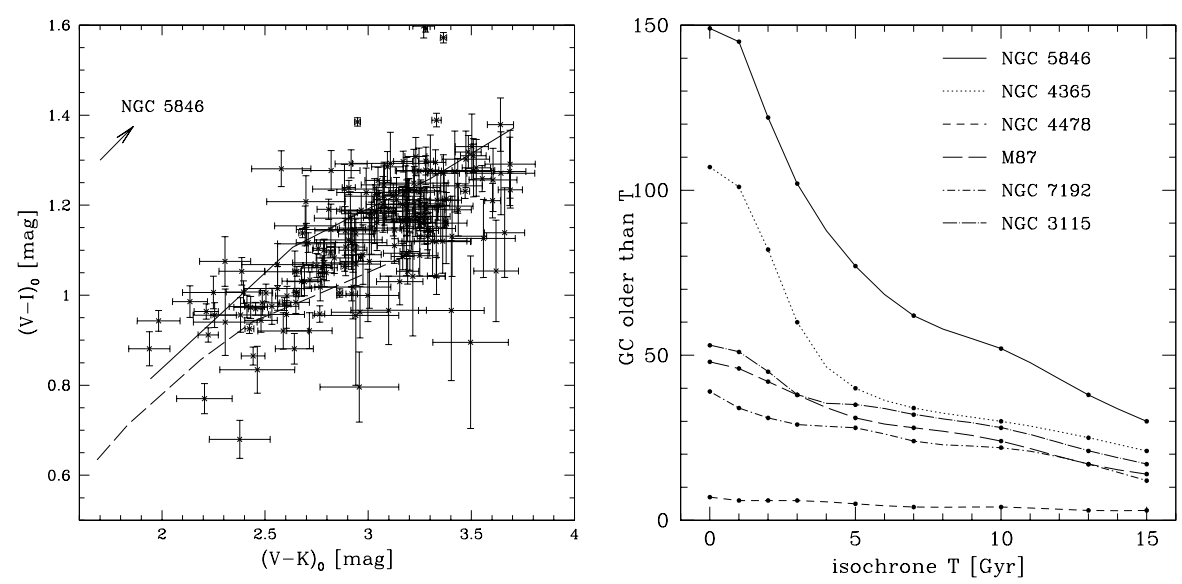

Figure 2. Left: $(V-I) v s .(V-K)$-color-color diagram for the NGC 5846 globular cluster system. The solid and dashed line mark the 15 Gyr and 2 Gyr isochrone, following the Bruzual \& Charlot SSP models (Bruzual 2000). Right: Cumulative age distribution in various galaxies derived with the above described procedure (see also Hempel \& Kissler-Patig (2004)).

- Simulate the color-color distributions for GCSs with a given age structure, e.g. a composition of 13 Gyr and 5 Gyr old objects. The number ratio between both populations may vary in $10 \%$ increments between a purely old or young population. In dependence on the availability of SSP isochrones we assume GC ages of 1, 1.5, 2, 3, 5, 7, 10, and 13 Gyr, respectively.

- Derive the cumulative age distribution of the simulated color distributions in the same way as for the observed systems.

- Compare the cumulative age distribution in the observed GCS with the complete set of simulated systems (different age and size for sub-populations) using a reduced $\chi^{2}$-test. The identification of a "best fitting" model allows us to set constraints on the age structure in the observed systems.

\section{Results}

This project aims at the detection of globular cluster sub-populations in early-type galaxies, considering various external parameters. For two selected galaxies, NGC 5846 and NGC 7192 the results are presented in Figure 3. The former is a member of a group of galaxies, whereas NGC 7192 represents isolated galaxies, accompanied by only 2 close neighbors. In case of NGC 5846 the derived age distribution is best matched by a mixed population of old (13 Gyr) and 10 Gyr younger objects. Hereby we note that for our purpose, the detection of age populations, an accuracy of 3 Gyr is more than sufficient. However, we need to discuss the surprisingly large fraction of intermediate age globular clusters. As described in Hempel \& Kissler-Patig (2004) photometric globular cluster surveys so far have been centered on the host galaxy, which leads to considerable spatial bias (see also Schweizer \& Seitzer 1998; Mihos \& Hibbard 1995). A more accurate determination of the relative sizes of sub-populations requires therefore spatially extended observations. Whereas in a larger group of galaxies interactions between its members are not unlikely, isolated galaxies like NGC 7192 will correspond much better to the picture of early-type galaxies as old and passively evolving galaxies. In agreement to that we find the cumulative age distribution dominated by an old population. At this point we do not distinguish between the possibility of a small fraction of intermediate age globular 

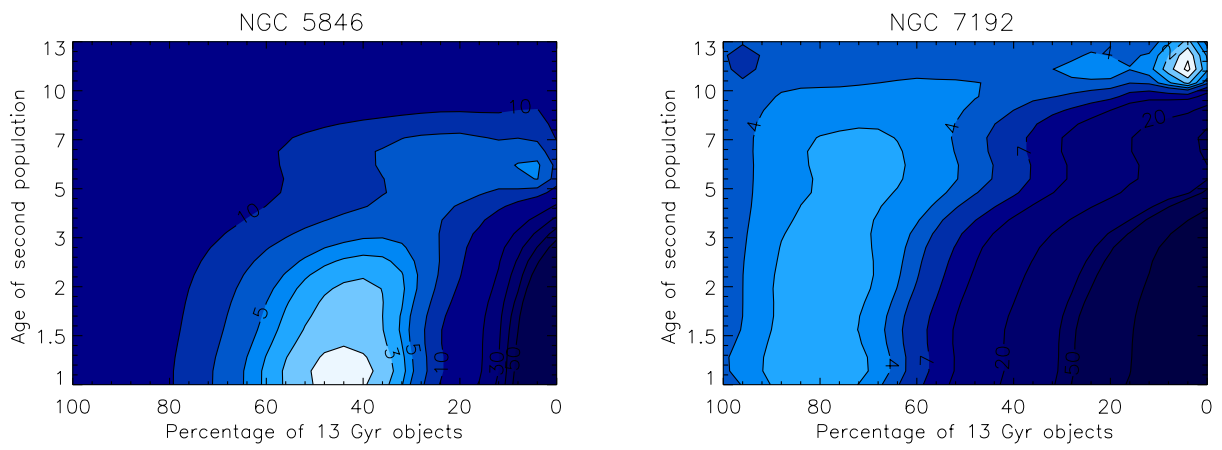

Figure 3. Result of the $\chi^{2}$-test for NGC 5846 (left) and NGC 7192 (right). The contours represent the $\chi^{2}$ values of the test. The best fitting model to the cumulative age distribution in NGC 5846 is clearly dominated by a population of intermediate age ( $\approx 1-2$ Gyr) globular clusters, whereas in NGC 7192 highest agreement between observation and model is obtained assuming either a purely 13 Gyr old population or a small contribution of second generation globular clusters with a highly uncertain age.

clusters or a system which is dominated by a second generation of globular clusters which is only slightly younger than the first generation (between 10 and 13 Gyr).

\section{Conclusions}

With the semi-numerical approach for age determination in globular cluster systems we are able to detect age sub-populations in early-type galaxies. Especially in case of the giant elliptical galaxies and their extremely rich globular cluster systems this will lead to a better understanding about their formation. For instance, the knowledge whether the observed blue colors of globular clusters are caused by very low metallicities or young/intermediate ages gives evidence for a possible accretion or stripping from dwarf galaxies or merger induced star formation. Although the data set needs to be expanded considerably we can already say that the major star formation epochs of early-type galaxies are far more diverse than previously thought.

\section{References}

Ashman, K.M. \& Zepf, S.E. 1998, Globular Cluster Systems, Cambridge University Press

Beasley, M.A., Sharples, R.M., Bridges, T.J. et al. 2000, MNRAS 318, 1249

Bruzual, A.G. 2000, private communication

Bruzual, A.G. \& Charlot, S. 2003, MNRAS 344, 1000

Chiosi, C. \& Carraro, G., 2000, MNRAS 335, 335

Gebhardt, K. \& Kissler-Patig, M. 1999, AJ 118, 1525

Hempel, M. \& Kissler-Patig, M. 2004, A\& $A$ 419, 863

Hilker, M., Infante, L. \& Richtler, T. 1999, AAS 138, 55

Hilker, M. 2003, Extragalactic Globular Cluster Systems, ESO Astrophysics Symp., eds. KisslerPatig, 173

Kauffman, G., White, S.D.M. \& Guiderdoni, B. 1993, MNRAS 264, 201

Kundu, A. \& Whitmore, B.C. 2001, $A J$ 121, 2950

Kundu, A. \& Whitmore, B.C. 2001, AJ 122, 1251

Maraston, C. 2005, MNRAS submitted

Vazdekis, A. 1999, ApJ 513, 224

Woodworth, S.C. \& Harris, W.E. 2000, AJ 119, 2699

Worthey, G. 1994, ApJS 95, 107 